\title{
The novel synthesized 6-fluoro-(3-fluorophenyl)-4-(3- methoxyanilino)quinazoline (LJJ-10) compound exhibits anti-metastatic effects in human osteosarcoma U-2 OS cells through targeting insulin-like growth factor-I receptor
}

\author{
KUAN-TIN CHEN $^{1 *}$, MANN-JEN HOUR $^{3 *}$, SHIH-CHANG TSAI ${ }^{2}$, JING-GUNG CHUNG $^{2}$, \\ SHENG-CHU KUO ${ }^{4}$, CHI-CHENG LU ${ }^{5}$, YU-JEN CHIU ${ }^{1}$, YI-HSUAN CHUANG ${ }^{1}$ and JAI-SING YANG ${ }^{1}$ \\ Departments of ${ }^{1}$ Pharmacology and ${ }^{2}$ Biological Science and Technology, ${ }^{3}$ School of Pharmacy, \\ ${ }^{4}$ Graduate Institute of Pharmaceutical Chemistry, China Medical University, Taichung 404; \\ ${ }^{5}$ Department of Life Sciences, National Chung Hsing University, Taichung 402, Taiwan, R.O.C.
}

Received March 15, 2011; Accepted May 12, 2011

DOI: $10.3892 /$ ijo.2011.1071

\begin{abstract}
Our previous study demonstrated that 6-fluoro(3-fluorophenyl)-4-(3-methoxyanilino)quinazoline (LJJ-10) possesses potential anticancer activity and exhibits greater antitumor effect than the other quinazoline compounds in human osteogenic sarcoma U-2 OS cells via in vitro screening. In this study, we focused on investigating the anti-metastatic activity and the signaling pathways involved in LJJ-10 action in U-2 OS cells. The results from wound healing and Boyden chamber transwell assays indicated that LJJ-10 exhibited an inhibitory effect on the migration and invasion of U-2 OS cells. LJJ-10 also inhibited matrix metalloproteinase-2 (MMP-2) and MMP-9 enzyme activities and caused a concentration-dependent decrease in protein levels by gelatin zymography assay and Western blot analysis, respectively. Meanwhile, LJJ-10 suppressed MMP-2 and MMP-9 mRNA levels in a concentration-dependent fashion after 12-h exposure in U-2 OS cells. Computational modeling showed that LJJ-10 is bound into the IGF-1R via hydrophobic interactions with $\mathrm{Leu}^{975}, \mathrm{Val}^{983}$, $\mathrm{Ala}^{1001}, \mathrm{Glu}^{1050}$ and $\mathrm{Met}^{1052}$ with one hydrogen bond between $6-\mathrm{F}$ and $\mathrm{Met}^{1052}$. LJJ-10 reduced the protein levels of $\mathrm{p}-\mathrm{JNK}$, p-p38, p-ERK, p-AKT and p-IGFR by Western blotting and these influences are concentration-dependent. Based on these observations, this study suggests that molecular targeting of the insulin-like growth factor-I receptor (IGF-1R) signaling
\end{abstract}

Correspondence to: Dr Jai-Sing Yang, Department of Pharmacology, China Medical University, No. 91, Hsueh-Shih Road, Taichung 40402, Taiwan, R.O.C.

E-mail: jaising@mail.cmu.edu.tw

*Contributed equally

Key words: LJJ-10, human osteosarcoma U-2 OS cells, antimetastasis, MMP-2 and MMP-9, IGF-1R signaling leads to the suppression of downstream MAPK/AKT signaling and downregulation of MMP-2 and -9 RNA levels and protein levels in LJJ-10-treated U-2 OS cells. Therefore, the inhibition of metastasis in human osteosarcoma cells by treatment with this novel agent, LJJ-10 may be a useful chemotherapeutic approach.

\section{Introduction}

Insulin-like growth factor-I receptor (IGF-1R) is an important receptor tyrosine kinase (RTK) on cell membrane surface (1). IGF-1R stimulates cell proliferation through ligands (IGF-1 or IGF-2). Upon binding of ligands, the IGF-1R becomes autophosphorylated on several tyrosine residues and activation of the mitogen-activated protein kinase (MAPK) and phosphoinositide 3-kinases (PI3K)/AKT downstream signaling (2). Also, IGF-1R signaling is important for cellular processes that are activated in tumor cells, including unmoral proliferation, metastasis and angiogenesis (3). Blocking of IGF-1R function has been shown to inhibit cell proliferation or metastasis, angiogenesis on breast tumor, lung tumor and colorectal tumor cells (4). Numerous studies have reported that the levels of IGF-1R, IGF-1 and IGF-2 were higher in human osteosarcoma cells than that in normal cells $(5,6)$. Many studies in therapeutic agents for osteosarcoma have focused on novel target therapies through induction of IGF-1R signaling, reduction of cell growth and anti-metastatic effects in osteogenic sarcoma $(5,7)$.

The clinical success of specific receptor tyrosine kinase inhibitors, such as gefitinib (Iressa), as therapeutic agents for human tumors has prompted substantial interest in development and clinical testing of these inhibitors for a broad range of malignancies (8). Therefore, novel agents are used for optimizing chemotherapy and it is reported that quinazoline derivatives have multiple biological activities, as receptor tyrosine kinase inhibitors (9). We designed the novel fluorinated compounds with a quinazoline core structure as anti-tumor candidates in order to increase their metabolic stability and be 
recognized by macromolecular recognition sites after entering human circulation (10). In this study, we synthesized a series of 6-fluoro-2-(3-fluorophenyl)-4-substituted anilinoquinazoline derivatives and assayed for cytotoxicity in vitro against seven types of cancer cell lines (10). Our previous result also showed that the novel compound, 6-fluoro-(3-fluorophenyl)4-(3-methoxyanilino) quinazoline (LJJ-10; Fig. 1) has greater cytotoxicity than other compounds in U-2 OS human osteosarcoma cells. In the present study, we focused on investigation of the molecular mechanisms in anti-metastasis in LJJ-10-treated human osteogenic sarcoma U-2 OS cells. Our results suggest that LJJ-10 might inhibit metastasis in U-2 OS cells through disrupting IGF-IR signaling in vitro.

\section{Materials and methods}

Chemicals and reagents. LJJ-10 was designed and synthesized by Dr Mann-Jen Hour and Dr Sheng-Chu Kuo (China Medical University, Taichung, Taiwan). Dimethyl sulfoxide (DMSO), McCoy's 5a medium, fetal bovine serum (FBS), L-glutamine, penicillin-streptomycin and trypsin-EDTA were obtained from Invitrogen Life Technologies (Carlsbad, CA, USA). Antibodies against phospho-AKT, phospho-JNK, phospho-ERK, phospho-p38, phospho-IGF-1R (Tyr 980) were purchased from Cell Signaling Technology Inc. (Danvers, MA, USA), and antibodies against AKT, JNK, ERK, p38, Actin, IGF-1R, MMP-2, MMP-9 and all peroxidase-conjugated secondary antibodies were purchased from Santa Cruz Biotechnology, Inc. (Santa Cruz, CA, USA).

Cell culture. The human osteosarcoma U-2 OS cell line was purchased from the Food Industry Research and Development Institute (Hsinchu, Taiwan). U-2 OS cells were cultured with 90\% McCoy's 5a medium and plated onto $75 \mathrm{~cm}^{2}$ tissue culture flasks with $2 \mathrm{mM}$ L-glutamine, 10\% FBS, $100 \mathrm{U} / \mathrm{ml}$ penicillin and $100 \mu \mathrm{g} / \mathrm{ml}$ streptomycin. All cells were grown at $37^{\circ} \mathrm{C}$ in a humidified atmosphere comprised of $95 \%$ air and $5 \% \mathrm{CO}_{2}(11)$.

Wound healing assay. U-2 OS cells were grown on 6-well dish plates to $100 \%$ confluent monolayer and then scratched to form a $100 \mu \mathrm{m}$ 'wound' using sterile pipette tips. The cells were then cultured in the presence or absence of LJJ-10 (0, 5, $10,20$ and $30 \mu \mathrm{M})$ in serum-free media for $24 \mathrm{~h}$. The images were recorded at 24-h intervals after scratching by using an Olympus photomicroscope. Cells were photographed under a phase-contrast microscope (x100) and the mean number of cells in the denuded zone area were calculated as previously described (12).

Transwell assay. The invasion of U-2 OS cells was measured using Matrigel-coated (BD Biosciences, San Jose, CA, USA) Transwell cell culture chambers $(8 \mu \mathrm{m}$ pore size; Millipore, Billerica, MA, USA) as previously described $(13,14)$. Cells were maintained in serum-free McCoy's 5a medium for $24 \mathrm{~h}$ before being trypsinized and re-suspended in serum-free medium. Cells were then placed in the upper chamber of the Transwell insert $\left(1 \times 10^{4}\right.$ cells/well) and treated with LJJ-10 (0, 5 , 10,20 and $30 \mu \mathrm{M}$ ) for $24 \mathrm{~h}$ in a humidified atmosphere with $95 \%$ air and $5 \% \mathrm{CO}_{2}$ at $37^{\circ} \mathrm{C}$. McCoy's 5a medium containing
$10 \%$ FBS was placed in the lower chamber. Invasive cells were fixed with $4 \%$ formaldehyde (Sigma-Aldrich Corp. St. Louis, MO, USA) and then stained with $2 \%$ crystal violet (Sigma-Aldrich Corp.). The non-invaded cells in the top well were removed with a cotton swab, and the invaded cells which penetrated through the Matrigel to the bottom wells were counted under a light microscope (x200). Each treatment of cells was assayed in duplicate, and three independent experiments were carried out (13).

Gelatin zymography. The activities of MMP-2 and MMP-9 were examined by gelatin zymography as described previously (13). Briefly, the U-2 OS cells $\left(2.5 \times 10^{5}\right.$ cells) were plated in 24-well culture plates and incubated in serum-free McCoy's 5a medium in the presence of LJJ-10 (5, 10, 20 and $30 \mu \mathrm{M}$ ) for $24 \mathrm{~h}$. The medium was collected and then separated by electrophoresis on $10 \%$ SDS-PAGE containing $0.1 \%$ gelatin (Sigma-Aldrich Corp.). After electrophoresis, the gels were soaked in $2.5 \%$ Triton X-100 in $\mathrm{ddH}_{2} \mathrm{O}$ twice for a total of $60 \mathrm{~min}$ at room temperature. Sample was incubated in substrate buffer $\left(50 \mathrm{mM}\right.$ of Tris $\mathrm{HCl}, 5 \mathrm{mM}$ of $\mathrm{CaCl}_{2}$, $0.02 \%$ of $\mathrm{NaN}_{3}$ and $1 \%$ of Triton $\mathrm{X}-100, \mathrm{pH} 8.0$ ) at $37^{\circ} \mathrm{C}$ for $24 \mathrm{~h}$. Bands corresponding to activity of MMP-2 and -9 were visualized by negative staining using $0.3 \%$ Coomassie blue in $50 \%$ methanol and $10 \%$ acetic acid (13).

Real-time PCR of MMP-2 and MMP-9. U-2 OS cells were cultured in 75-T flasks and treated with LJJ-10 at final concentration of 10,20 and $30 \mu \mathrm{M}$ for $12 \mathrm{~h}$. Cells were harvested and total RNA was extracted with the Qiagen RNeasy Mini Kit (Qiagen, Inc, Valencia, CA, USA). RNA samples were reverse-transcribed at $42^{\circ} \mathrm{C}$ with High Capacity cDNA Reverse Transcription Kit for 30 min according to the protocol of the supplier (Applied Biosystems, Carlsbad, CA, USA). Quantitative PCR conditions were as follows: $2 \mathrm{~min}$ at $50^{\circ} \mathrm{C}, 10 \mathrm{~min}$ at $95^{\circ} \mathrm{C}$, and 40 cycles of $15 \mathrm{sec}$ at $95^{\circ} \mathrm{C}$; $1 \mathrm{~min}$ at $60^{\circ} \mathrm{C}$ using $1 \mu \mathrm{l}$ of the cDNA reverse-transcribed as described above, 2X SYBR Green $\mathrm{P} \mu \mathrm{CR}$ Master Mix (Applied Biosystems) and $200 \mathrm{nM}$ forward (F) and reverse (R) primers (homo MMP-2-F: CCCCAGACAGGTGATCTTGAC; homo MMP-2-R: GCTTGCGAGGGAAGAAGTTG; homo MMP-9-F: CGCTGGGCTTAGATCATTCC; homo MMP-9-R: AGGTTGGATACATCACTGCATTAGG; homo GAPDH-F ACACCCACTCCTCCACCTTT; homo GAPDH-R TAGCCA AATTCGTTGTCATACC). Applied Biosystems 7300 Realtime PCR System was used for each assay in triplicate, and expression fold-changes were derived using the comparative CT method as described previously $(13,15)$.

Molecular modeling analysis. The three dimensional crystal structure of the IGF-1R was downloaded from RCSB Protein Data Bank website (http://www.rcsb.org/pdb). Automated docking was then carried out. The LigandFit within the software package Discovery Studio 2.5 (Accelrys Inc., San Diego, CA, USA) was used to evaluate and predict the in silico binding free energy of the inhibitors within the macromolecules. The protocol was used to prepare 2oj9 protein structure including standardize atom names, by inserting missing atoms in residues and removing alternate conformations, inserting missing loop regions based on SEQRES data, optimizing 
short and medium size loop regions with Looper Algorithm, minimizing remaining loop regions, and calculating $\mathrm{pK}$ and protonate structure. A binding pocket of the native ligand was selected as the binding site for the study. Following typing of the receptor model with the CHARMm forcefield, the binding site was identified by the LigandFit flood-filling algorithm. This docking protocol employed total ligand flexibility whereby the final ligand conformations were determined by the Monte Carlo conformation search method set to a variable number of trial runs. The docked ligands were further refined using in situ ligand minimization with the Smart Minimizer algorithm. Each minimization was carried out in two steps, first using steepest descent minimization for 200 cycles and then using conjugate gradient minimization, until the average gradient fell below $0.01 \mathrm{kcal} / \mathrm{mol}$. All atoms within $6.0 \AA$ of the inhibitor were allowed to relax during the minimization, whereas those atoms beyond $6.0 \AA$ were held rigid. At last, Dock score was used to estimate the binding free energies of the ligands. Among the docked conformations, the pose with highest value of Dock score was selected for the calculation of binding free energy $(\Delta \mathrm{Gb})$ and inhibition constant $(\mathrm{Ki})$ (16).

Western blot analysis. U-2 OS cells $\left(1.0 \times 10^{7}\right.$ cells) were cultured in 75-T flasks and exposed to various concentrations of LJJ-10 (10, 20 and $30 \mu \mathrm{M})$ for $3 \mathrm{~h}$. Cells were harvested and resuspended in the PRO-PREP ${ }^{\mathrm{TM}}$ protein extraction solution (iNtRON Biotechnology, Seongnam, Gyeonggi-Do, Korea). The collected cells were centrifuged at $13,000 \mathrm{~g}$ for $10 \mathrm{~min}$ at $4^{\circ} \mathrm{C}$ to remove cell debris, and the supernatant was collected for determination of total protein concentration using a Bio-Rad protein assay kit (Hercules, CA, USA) with bovine serum albumin (BSA) as the standard. Protein samples $(30 \mu \mathrm{g})$ were electrophoresis by $10-15 \%$ sodium dodecyl sulfate polyacrylamide gel electrophoresis (SDS-PAGE) and transferred onto nitrocellulose membranes (Invitrogen). Membrane was then blocked with in PBST (0.1\% Tween-20 in PBS) plus $5 \%$ powdered non-fat milk for $1 \mathrm{~h}$, and incubated overnight at $4^{\circ} \mathrm{C}$ with each of the following specific primary antibodies (anti-phospho-AKT, anti-phospho-JNK, anti-phospho-ERK, anti-phospho-p38, anti-phospho-IGF-1R (Tyr980), anti-AKT, anti-JNK, anti-ERK, p38, anti-actin, anti-IGF-1R, antiMMP-2, anti-MMP-9 antibodies). The membranes were washed with PBST three times for $10 \mathrm{~min}$ and incubated with HRP conjugated secondary IgG antibody (horseradish peroxidase-conjugated goat anti-rabbit and goat anti-mouse) for $1 \mathrm{~h}$ at room temperature. Bands were detected by enhanced chemiluminescence with ECL reagents (Amersham Pharmacia, Buckinghamshire, UK) and exposed to X-OMAT AR film (Eastman Kodak, Rochester, NY, USA). The autoradiograms were scanned on UMAX PowerLook Scanner (UMAX Technologies, Fremont, CA, USA) with Photoshop software (Adobe Systems, Seattle, WA, USA). All results were performed in three independent experiments $(17,18)$.

Statistical analysis. The data are expressed as the mean \pm SEM from at least three separate experiments. Statistical calculations of the data were performed by using one-way ANOVA followed by Bonferroni's test for multiple comparisons. A $\mathrm{p}<0.05$ was considered statistically significant.

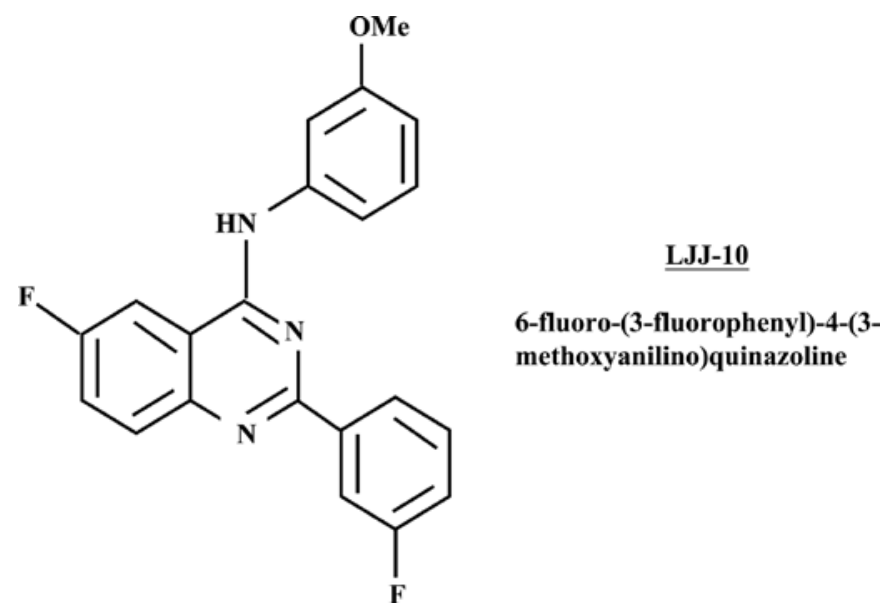

Figure 1. The chemical structure of LJJ-10.

\section{Results}

LJJ-10 inhibits migration and invasion of U-2 OS cells. The inhibition of cell migration by LJJ-10 was examined by using Wound healing assay. As shown in Fig. 2A, LJJ-10 (5-30 $\mu \mathrm{M})$ significantly inhibited cell migration in a concentrationdependent manner; the percentage of inhibition ratio was 27-78\%. The inhibition of cell invasion by LJJ-10 was examined by using Matrigel-coated Transwell assay. As shown in Fig. 2B, LJJ-10 (5-30 $\mu \mathrm{M})$ significantly inhibited cell invasion in a concentration-dependent manner; the percentage of inhibition ratio was $20-80 \%$. We determined the cytotoxicity of LJJ-10 using MTT assay. LJJ-10 did not affect cell viability at $5-30 \mu \mathrm{M}$ of $24-\mathrm{h}$ treatment (data not shown). On the other hand, the $\mathrm{EC}_{50}$ is $75.32 \pm 3.25 \mu \mathrm{M}$ for $24 \mathrm{~h}$ in LJJ-10-treated $\mathrm{U}-2$ OS cells. Our results suggest that LJJ-10 inhibited the effects of cell migration and invasion in U-2 OS cells. Also, the inhibitory effects of LJJ-10 on cell migration and invasion are independent of cellular cytotoxicity.

LJJ-10 suppresses MMP-2/MMP-9 enzyme activities and protein levels. Matrix metalloproteinase-2 and -9 (MMP-2 and MMP-9) are important pre-requisite for tumor invasion and metastasis in human osteosarcoma cells (15). The gelatin zymography was used to analyze the effects of LJJ-10 on MMP-2 and MMP-9 activities for 24-h treatment. As shown in Fig. 3A, LJJ-10 (5-30 $\mu \mathrm{M})$ significantly inhibited MMP-2 and MMP-9 enzyme activities in a concentration-dependent manner. These results were also confirmed by Western blot analysis as can be seen in Fig. 3B. LJJ-10 decreased the protein levels of MMP-2 and MMP-9 (Fig. 3B). Our results suggest that LJJ-10 inhibited invasion and migration in U-2 OS cells through decreasing the enzyme activity and protein levels of MMP-2 and MMP-9.

LJJ-10 decreases MMP-2/MMP-9 mRNA levels. Real-time PCR analysis was performed to determine whether the inhibition of MMP-2 and MMP-9 protein levels and activities by LJJ-10 was due to decreased the levels of mRNA. As shown in Fig. 4, the 12-h treatment of U-2 OS cells with LJJ-10 (10, 20 and $30 \mu \mathrm{M}$ ) led to a decrease in mRNA levels of MMP-9 
A

\section{Cell migration assay}
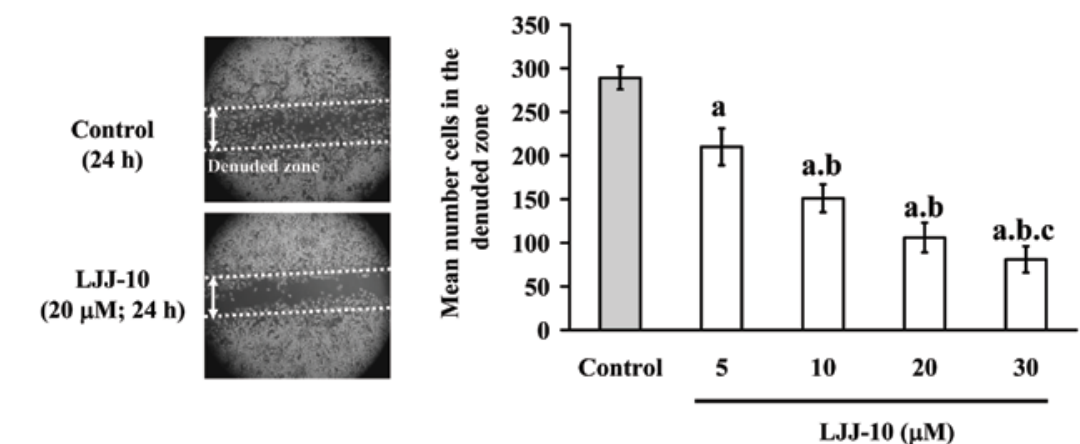

B

\section{Cell invasion assay}
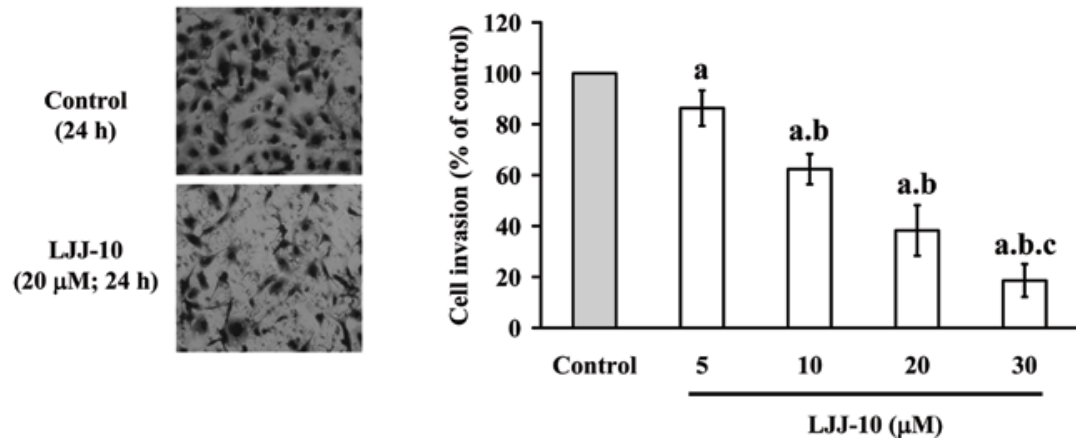

Figure 2. LJJ-10 inhibits the migration and invasion of U-2 OS cells in vitro. Cells were treated with 0, 5, 10, 20 and $30 \mu \mathrm{M}$ of LJJ-10 for $24 \mathrm{~h}$. (A) Cell motility was determined by wound healing assay. Cell morphology was micro-photographed at magnification x100 (left panel) and the mean number cells in the denuded zone area are calculated (right panel). (B) Invaded cells through the Matrigel to the lower surface of the filter those were stained with crystal violet and were photographed under a light microscope at magnification x200 (left panel). Quantification of cells in the lower chamber was performed by counting the cells (right panel). Data are presented as the mean $\pm \mathrm{SD}(\mathrm{n}=3)$. a, $\mathrm{p}<0.05$, is significantly different compared with the DMSO-treated control; $b$ and c, p $<0.05$, are significantly different compared with 5 and $10 \mu \mathrm{M}$ of LJJ-10 treatment, respectively, by one-way ANOVA followed by Bonferroni's test for multiple comparisons.
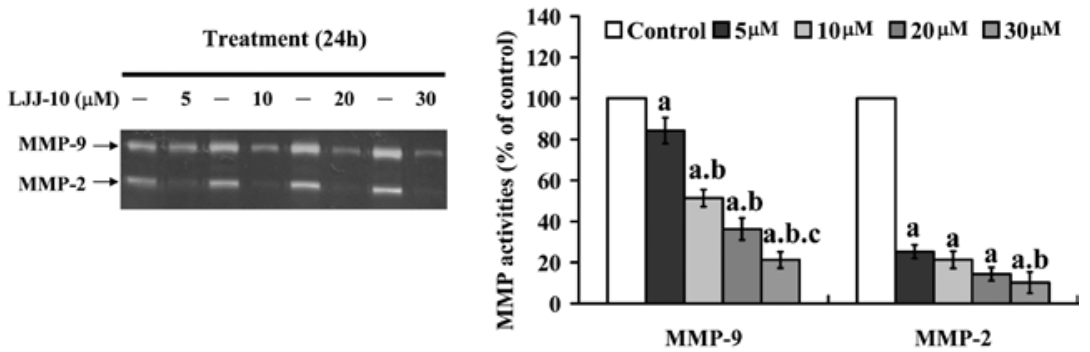

B

$\underline{\text { Western Blot assay }}$
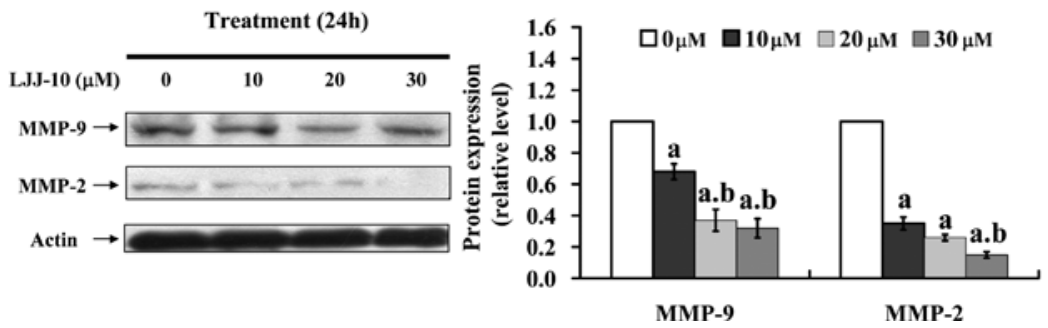

Figure 3. LJJ-10 affected MMP-2 and MMP-9 activities and protein levels in U-2 OS cells. Cells were incubated with 0,5, 10, 20 and $30 \mu \mathrm{M}$ of LJJ-10 for 24 h. (A) For MMP-2 and MMP-9 activity assay, the culture media for treated and non-treated cells were harvested and separated by gelatin zymography (left panel) and the ratio of MMP-2 and MMP-9 activities were quantified (right panel). (B) The proteins were prepared and analyzed by Western blotting using antibodies against MMP-2 and MMP-9. The results are shown in the left panel and protein levels were quantified by image analysis (right panel) as described in Materials and methods. Data are presented as the mean $\pm \mathrm{SD}(\mathrm{n}=3)$. a, $\mathrm{p}<0.05$, is significantly different compared with the DMSO-treated control; $\mathrm{b}$ and $\mathrm{c}, \mathrm{p}<0.05$, are significantly different compared with 5 and $10 \mu \mathrm{M}$ of LJJ-10 treatment, respectively, by one-way ANOVA followed by Bonferroni's test for multiple comparisons. 


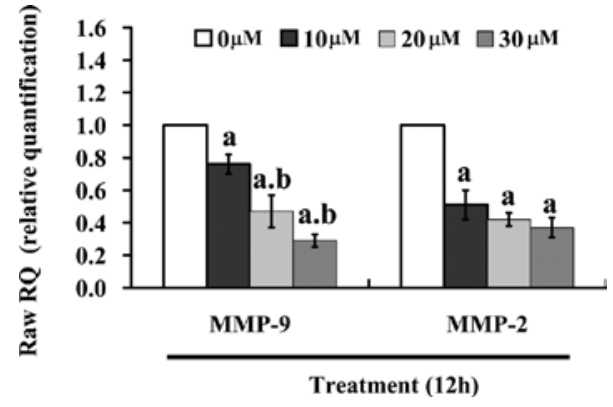

Figure 4. Effects of LJJ-10 on MMP-2 and MMP-9 mRNA levels in U-2 OS cells. The total RNA was extracted from each treatment of LJJ-10 $(0,10,20$ and $30 \mu \mathrm{M})$ in $\mathrm{U}-2$ OS cells for $12 \mathrm{~h}$. RNA samples were reverse-transcribed into cDNA and quantified with real-time PCR as described in Materials and methods. The ratios of MMP-2 and MMP-9 to GAPDH mRNA are presented. Data are presented as the mean $\pm S D(n=3)$. a, $p<0.05$, is significantly different compared with the DMSO-treated control; $b, p<0.05$, is significantly different with $10 \mu \mathrm{M}$ of LJJ-10 treatment by one-way ANOVA followed by Bonferroni's test for multiple comparisons. and MMP-2. Our results indicate that LJJ-10 inhibited the protein levels and activities of MMP-2 and MMP-9 through the regulation of transcription levels.

Computational modeling and docking results. It was reported that the quinazoline ring system is a template for receptor tyrosine kinase inhibitors (9). We have designed and synthesized a novel quinazoline derivative LJJ-10 which is possibly a novel anti-IGF-1R agent. To predict the major target site of LJJ-10, the LigandFit within the software package Discovery Studio 2.5 was used to ensure the target of LJJ-10. As shown in Fig. 5A, LJJ-10 is bound into the IGF-1R via hydrophobic interactions with $\mathrm{Leu}^{975}$, $\mathrm{Val}^{983}$, $\mathrm{Ala}^{1001}$, Glu ${ }^{1050}$ and $\mathrm{Met}^{1052}$ with one hydrogen bond between 6-F and $\mathrm{Met}^{1052}$. After surveying the PDB bank, the 2oj9, IGF-1R kinase domain complexes with a benzimidazole inhibitor (BMI, (3-(5-imidazol-1-yl-7methyl-1H-benzimidazol-2-yl)-4-(pyridin-2-ylmethylamino)-

$\mathbf{A}$

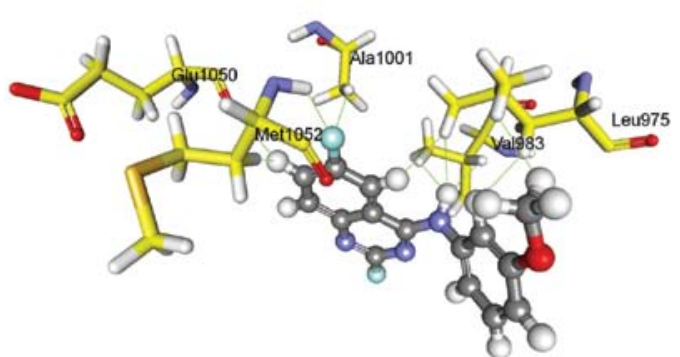

B

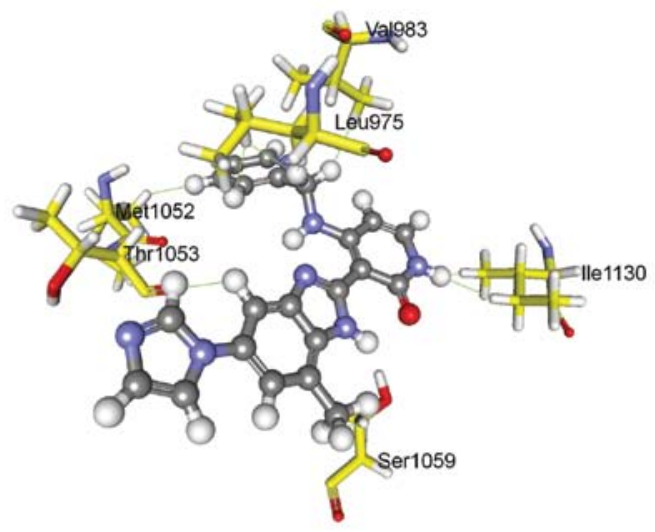

C

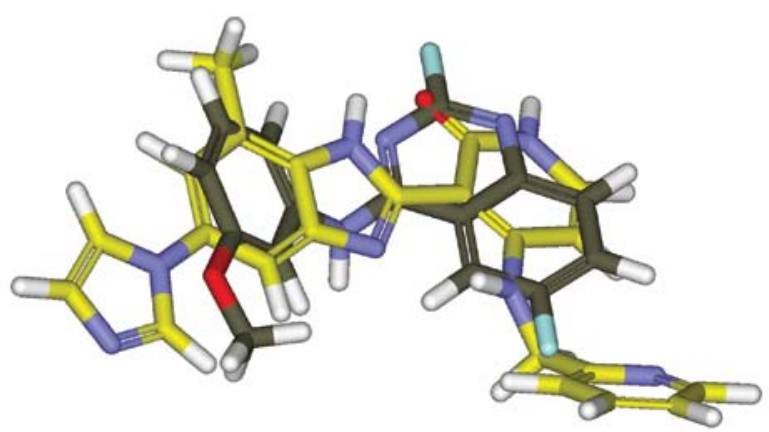

LJJ-10

6-fluoro-(3-fluorophenyl)-4-(3methoxyanilino)quinazoline

\section{BMI}

(3-(5-imidazol-1-yl-7-methyl-1Hbenzimidazol-2-yl)-4-(pyridin-2ylmethylamino)-1H-pyridin-2one)

Figure 5. Predicted binding form of LJJ-10 with IGF-1R by molecular modeling analysis. (A) Model of HMJ-10 and IGF-1R (2oj9). LJJ-10 (ball and stick, colored by elements) is bound into the IGF-1R via hydrophobic interactions with Leu ${ }^{975}$, Val ${ }^{983}$, Ala ${ }^{1001}$, Glu ${ }^{1050}$ and Met ${ }^{1052}$, in addition with one hydrogen bond between 6-F and Met ${ }^{1052}$. The binding amino acids are shown as yellow sticks and labeled. (B) Model of BMI and IGF-1R (2oj9). BMI (3-(5-imidazol-1-yl-7methyl-1H-benzimidazol-2-yl)-4-(pyridin-2-ylmethylamino)-1H-pyridin-2-one, ball and stick, colored by elements) is bound into the IGF-1R via hydrophobic interactions with $\mathrm{Leu}^{975}$, Val ${ }^{983}$, $\mathrm{Met}^{1052}$, Thr ${ }^{1053}$, $\mathrm{Ser}^{1059}$ and $\mathrm{Ile}^{1130}$. The binding amino acids are shown as yellow sticks and labeled. (C) Superimposed BMI (shown as yellow stick) and LJJ-10 (shown as grey stick). 
Table I. The ligand scoring for LJJ-10 and BMI.

\begin{tabular}{lccccr}
\hline Name & $\begin{array}{c}\text { LigScore1 } \\
\text { Dreiding }\end{array}$ & $\begin{array}{c}\text { LigScore2 } \\
\text { Dreiding }\end{array}$ & $(-)$ PLP1 & (-) PLP2 & $\begin{array}{l}\text { (-) PMF } \\
\text { score }\end{array}$ \\
\hline LJJ-10 & 2.84 & 4.87 & 62.77 & 58.48 & 61.6 \\
BMI & 2.34 & 4.97 & 68.81 & 55.9 & 62.43 \\
\hline
\end{tabular}

LigScore1 and LigScore 2 are fast, simple, scoring functions for predicting receptor-ligand binding affinities. LigScore1_Dreiding = -0.3498$0.04673^{*} \mathrm{vdW}+0.1653^{*} \mathrm{C}+$ pol-0.001132 ${ }^{*} \mathrm{TotPol}^{\wedge} 2$. LigScore2_Dreiding $=1.539-0.07622^{*} \mathrm{vdW}+0.6501^{*} \mathrm{C}+\_$pol-0.0000782 ${ }^{*} \mathrm{BuryPol}^{\wedge} 2 . \mathrm{In}$ the PLP1 function, each non-hydrogen ligand or non-hydrogen receptor atom is assigned a PLP atom type. In the PLP2 function, PLP atom typing remains the same as in PLP1. In addition, an atomic radius is assigned to each atom except for hydrogen. The PMF scoring functions were developed based on statistical analysis of the 3D structures of protein-ligand complexes. Candidate ligand poses were evaluated and prioritized according to the DockScore function (Discovery Studio 2.5, http://accelrys.com/products/discovery-studio/).

$\mathbf{A}$

$\underline{\text { IGF-1R protein levels }}$
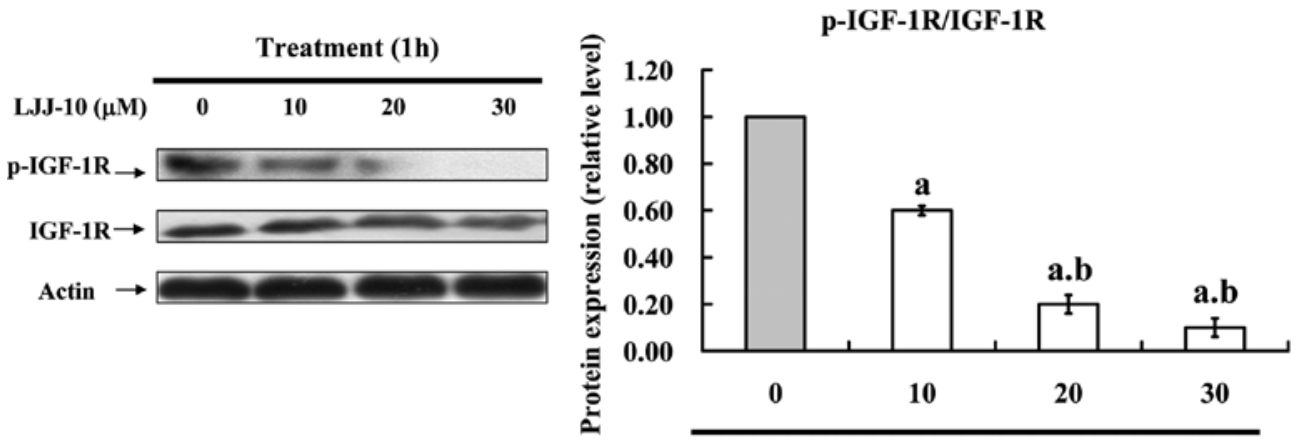

LJJ-10 ( $\mu \mathrm{M})$

B

$\underline{\text { MAPK/AKT protein levels }}$
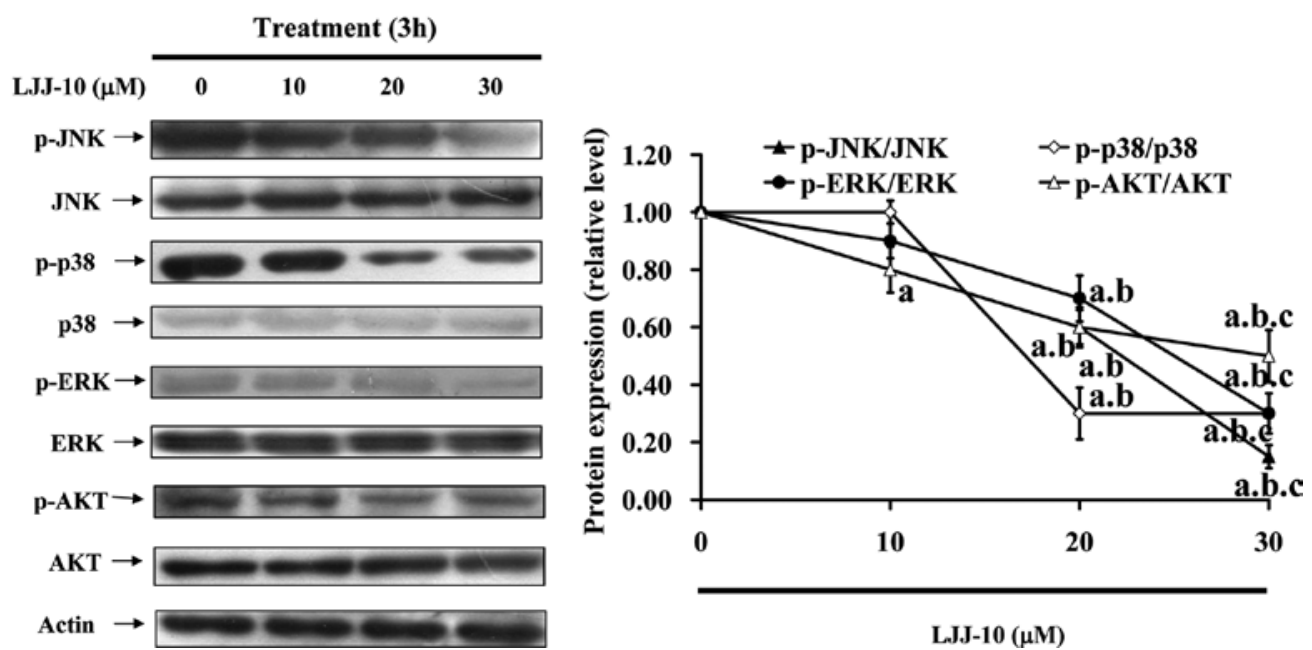

Figure 6. Effect of LJJ-10 on IGF-1R and downstream protein levels in U-2 OS cells. (A) Cells were treated with LJJ-10 (0, 10, 20 and $30 \mu \mathrm{M})$ for $1 \mathrm{~h}$. The protein levels of phosphorylated IGF-1R (Tyr 980) and IGF-1R were analyzed by Western blotting (left panel) and protein levels were quantified by image analysis (right panel). (B) The protein levels of p-JNK, JNK, p-p38, p38, p-ERK, ERK, p-AKT and AKT were analyzed by Western blotting (left panel) and protein levels were quantified by image analysis (right panel). Data are presented as the mean $\pm \mathrm{SD}(\mathrm{n}=3)$. a, $\mathrm{p}<0.05$, is significantly different compared with the DMSO-treated control; b and c, p $<0.05$, are significantly different with 10 and $20 \mu \mathrm{M}$ of LJJ-10 treatment, respectively, by one-way ANOVA followed by Bonferroni's test for multiple comparisons.

1H-pyridin-2-one), was downloaded (Fig. 5B). The LJJ-10 was docked into the binding site of BMI in 2oj9 and our models were scored using several functions, LigScore, PLP, PMF and DockScore. Because BMI is reported as a strong IGF-1R inhibitor and the structure conformation is somewhat similar to LJJ-10 (Fig. 5), BMI was used as a positive control in this simulation (16). Scores of the docked ligands are tabulated in Table I. The docking results show the scores of LJJ-10 were 


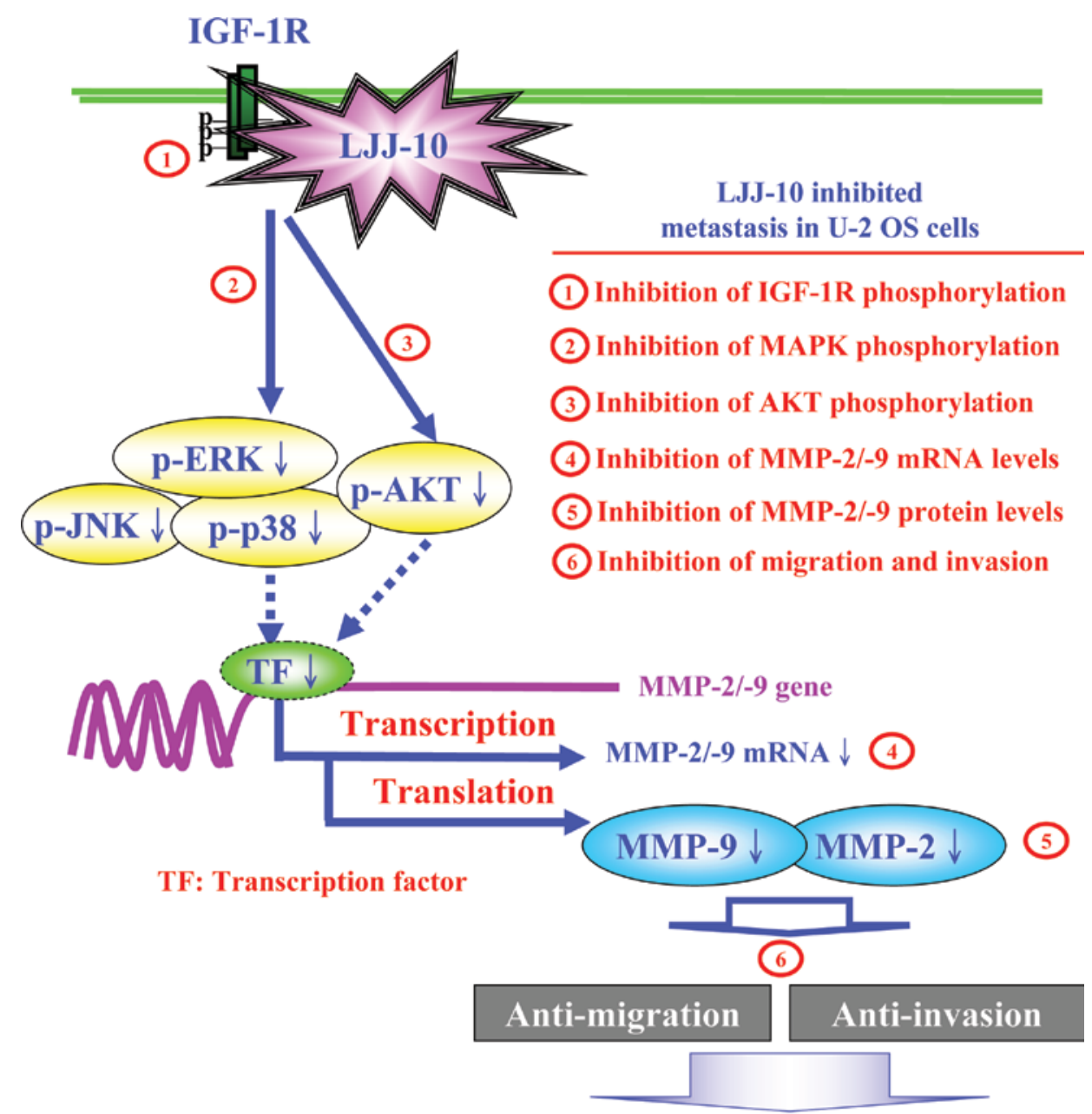

Anti-metastasis

Figure 7. LJJ-10 participates in the anti-metastatic molecular mechanisms of human osteosarcoma U-2 OS cells.

close to that of BMI (Fig. 5C). Consequently, we suggest that LJJ-10 interacts well with IGF-1R, and the IGF-1R should be a target of LJJ-10. The docking results show that there is a positive correlation between the simulation and our experimental data.

LJJ-10 reduced the protein levels of $p-I G F R, p-J N K, p-p 38$, $p$-ERK and $p-A K T$. We investigated the effects of LJJ-10 on IGF-1R and phospho-IGF-1R protein levels in U-2 OS cells by examining the IGF-1R phosphorylation states. As shown in Fig. 6A, we determined U-2 OS cells after exposure to LJJ-10 $(10,20$ and $30 \mu \mathrm{M})$ for $1 \mathrm{~h}$. Western blot analysis of IGF-1R phosphorylation with the antibody against p-IGF1RTyr980 showed that LJJ-10 reduced the protein level of p-IGF-1RTyr980 and these influences are dose-dependent. To illuminate the possible downstream signaling pathways in LJJ-10-treated U-2 OS cells, we evaluated the related protein levels in AKT and MAPK (JNK, p38 and ERK) signaling pathways by Western blotting. As shown in Fig. 6B, the cells were treated with $\operatorname{LJJ}-10(10,20$ and $30 \mu \mathrm{M})$ for $3 \mathrm{~h}$. The results showed that LJJ-10 reduced the protein levels of p-JNK, p-p38, p-ERK, p-AKT and the influence is concentration-dependent. Our results suggest that LJJ-10 blocks phosphorylation of IGF-1R, which led to inhibition of the downstream MAPK and AKT signaling pathways in U-2 OS cells.

\section{Discussion}

Interference with receptor tyrosine kinase provides a novel approach toward tumor therapy agents. Quinazoline derivatives are potential receptor tyrosine kinase inhibitors $(9,16)$. Several successful strategies for the inhibition of metastasis have been effectively demonstrated in preclinical and clinical settings. Gefitinib (Iressa) is a clinical success of selective kinase inhibitor; and improved effectiveness of first-, secondline and maintenance therapeutic regimens in non-small cell lung cancer $(8,19,20)$. Although Gefitinib (Iressa) have antimetastasis actions in clinical treatment, there are limitations to their use because of toxic side effects and drug resistance $(21,22)$. LJJ-10, a quinazoline compound was designed and synthesized for a promising anti-metastasis compound in our laboratory. Our result previously demonstrated that the LJJ-10 has greater cytotoxicity than the other compounds in human osteogenic sarcoma U-2 OS cells. LJJ-10 induced significant concentration-dependent growth inhibition and apoptotic cell death. The $\mathrm{EC}_{50}$ value of LJJ-10 was lower in human osteogenic sarcoma U-2 OS cells than in normal human fetal 
osteoblastic hFOB cells (data not shown). Our results suggest that LJJ-10 could be an efficacious and safer anti-tumor agent for treatment of human osteogenic sarcoma. In this study, we focused on investigating the anti-metastasis activity and the signaling pathway of LJJ-10-treated U-2 OS cells in vitro. Our results suggest that low concentration of LJJ-10 $(5-30 \mu \mathrm{M})$ significantly inhibits cell migration and invasion in U-2 OS cells (Fig 2). The $\mathrm{EC}_{50}$ is $75.32 \pm 3.25 \mu \mathrm{M}$ for $24 \mathrm{~h}$ in LJJ-10 treated U-2 OS cells, and LJJ-10 did not affect cell viability at 5-30 $\mu \mathrm{M}$ of $24-\mathrm{h}$ treatment. Our results suggest that the inhibitory effects of LJJ-10 on cell migration and invasion are independent of cellular cytotoxicity. LJJ-10 might be a useful anti-metastasis agent.

Insulin-like growth factor (IGF)-mediated signaling is involved in bone homeostasis and osteogenesis (23). Consistent expression of IGF-1R, IGF-1, and IGF-2 in osteosarcoma cell lines and patient samples has been reported $(24,25)$. Blocking IGF-mediated growth in osteosarcoma can inhibit tumor growth and metastasis in murine osteosarcoma $(5,26,27)$. In vivo study also demonstrated that tumor growth in xenografts can be inhibited with anti-IGF-1R antibody (5). In this study, we first used molecular modeling (Fig.5), and Western blot analysis (Fig. 6A). Our results suggest that LJJ-10 is an IGF-1R inhibition agent. Both IGF-1 and IGF-2 bind to IGF-1R, which leads to autophosphorylation of the receptor. When IGF-1R is phosphorylated, insulin receptor substrates 1 (IRS1) and the Src homology collagen-like adaptor protein (Shc) can turn on downstream intracellular signaling cascades as MAPK and AKT pathways $(28,29)$. Activation of MAPK and AKT results in enhanced tumor cell proliferation, migration, and survival (30). In our study, we demonstrated that $10-30 \mu \mathrm{M}$ of LJJ-10 significantly inhibited cell migration (Fig. 2A) and invasion (Fig. 2B) and inhibited MMP-2 and MMP-9 enzyme activities (Fig. 3A). LJJ-10 significantly inhibited MMP-2 and MMP-9 mRNA levels in U-2 OS cells (Fig. 4). It has been reported that the MMP-2 and MMP-9 promoters contain several transcription-factor-binding motifs that can affect its mRNA transcription, including AP-1, NF- $\kappa B$, Sp1 and p53 $(31,32)$. Our results suggested that LJJ-10 represses MMP-2 and MMP-9 transcription through inhibiting activation of transcription factors such as AP-1, NF- $\mathrm{kB}$, $\mathrm{Sp} 1$ and p53 in U-2 OS cells. As shown in Fig. 6B, LJJ-10 caused a decrease in the protein levels of p-JNK, p-p38, p-ERK, p-AKT in U-2 OS cells. Based on the observations, we suggest that LJJ-10 inhibited U-2 OS cell metastasis through triggering IGF-1R inhibition and downstream MAPK and AKT signaling pathways in U-2 OS cells.

Collectively, we have outlined the molecular mechanism and the overall possible signaling pathways for LJJ-10-inhibited metastasis in U-2 OS cells (Fig. 7). The proposed signal pathways of LJJ-10 exhibit anti-metastatic effects trigger IGF-1R and downstream signaling inhibition in human osteosarcoma U-2 OS cells. LJJ-10 may be an antiosteosarcoma drug candidate.

\section{Acknowledgments}

This study was supported by a research grant from the National Science Council of the Republic of China (NSC 99-2320-B-039-013-MY3; NSC 97-2320-B-039-004-MY3) and a grant from China Medical University, Taichung, Taiwan (CMU99-S-34).

\section{References}

1. Moro-Sibilot D, Coudurier M and Lantuejoul S: Targeting insulin-like growth factors in the treatment of cancer. Rev Mal Respir 27: 959-963, 2010.

2. Maines MD: Potential application of biliverdin reductase and its fragments to modulate insulin/IGF-1/MAPK/PI3-K signaling pathways in therapeutic settings. Curr Drug Targets 11: 1586-1594, 2010.

3. Zhang D, Samani AA and Brodt P: The role of the IGF-I receptor in the regulation of matrix metalloproteinases, tumor invasion and metastasis. Horm Metab Res 35: 802-808, 2003.

4. Miyamoto S, Yano K, Sugimoto S, et al: Matrix metalloproteinase-7 facilitates insulin-like growth factor bioavailability through its proteinase activity on insulin-like growth factor binding protein 3. Cancer Res 64: 665-671, 2004.

5. Kolb EA, Kamara D, Zhang W, et al: R1507, a fully human monoclonal antibody targeting IGF-1R, is effective alone and in combination with rapamycin in inhibiting growth of osteosarcoma xenografts. Pediatr Blood Cancer 55: 67-75, 2010.

6. Herzlieb N, Gallaher BW, Berthold A, Hille R and Kiess W: Insulin-like growth factor-I inhibits the progression of human U-2 OS osteosarcoma cells towards programmed cell death through interaction with the IGF-I receptor. Cell Mol Biol 46: 71-77, 2000.

7. Toretsky JA and Gorlick R: IGF-1R targeted treatment of sarcoma. Lancet Oncol 11: 105-106, 2010.

8. Armour AA and Watkins CL: The challenge of targeting EGFR: experience with gefitinib in nonsmall cell lung cancer. Eur Respir Rev 19: 186-196, 2010.

9. De Oliveira AN, Bocca CC, Carvalho JE, et al: New substituted 4-arylaminoquinazolines as potent inhibitors of breast tumor cell lines: in vitro and docking experiments. Eur J Med Chem 45: 4339-4342, 2010.

10. Hour MJ, Yang JS, Lien JC, Kuo SC and Huang LJ: Synthesis and cytotoxicity of 6-pyrrolidinyl-2-(2-substituted phenyl)4-quinazolinones. J Chin Chem Soc 54: 785-790, 2007.

11. Huang WW, Chiu YJ, Fan MJ, et al: Kaempferol induced apoptosis via endoplasmic reticulum stress and mitochondriadependent pathway in human osteosarcoma U-2 OS cells. Mol Nutr Food Res 54: 1585-1595, 2010.

12. Ho YT, Yang JS, Li TC, et al: Berberine suppresses in vitro migration and invasion of human SCC-4 tongue squamous cancer cells through the inhibitions of FAK, IKK, NF-kappaB, u-PA and MMP-2 and -9. Cancer Lett 279: 155-162, 2009.

13. Chen YY, Chiang SY, Lin JG, et al: Emodin, aloe-emodin and rhein inhibit migration and invasion in human tongue cancer SCC-4 cells through the inhibition of gene expression of matrix metalloproteinase-9. Int J Oncol 36: 1113-1120, 2010.

14. Lu KW, Chen JC, Lai TY, et al: Gypenosides inhibits migration and invasion of human oral cancer SAS cells through the inhibition of matrix metalloproteinase-2/ -9 and urokinaseplasminogen by ERK1/2 and NF-kappa B signaling pathways. Hum Exp Toxicol (In press).

15. Chiang JH, Yang JS, Ma CY, et al: Danthron, an anthraquinone derivative, induces DNA damage and caspase cascades-mediated apoptosis in SNU-1 human gastric cancer cells through mitochondrial permeability transition pores and Bax-triggered pathways. Chem Res Toxicol 24: 20-29, 2011.

16. Velaparthi U, Wittman M, Liu P, et al: Discovery and initial SAR of 3-(1H-benzo[d]imidazol-2-yl)pyridin-2 $(1 \mathrm{H})$-ones as inhibitors of insulin-like growth factor 1-receptor (IGF-1R). Bioorg Med Chem Lett 17: 2317-2321, 2007.

17. Yang MD, Lai KC, Lai TY, et al: Phenethyl isothiocyanate inhibits migration and invasion of human gastric cancer AGS cells through suppressing MAPK and NF-kappaB signal pathways. Anticancer Res 30: 2135-2143, 2010.

18. Lu CC, Yang JS, Huang AC, et al: Chrysophanol induces necrosis through the production of ROS and alteration of ATP levels in J5 human liver cancer cells. Mol Nutr Food Res 54: 967-976, 2010.

19. Zampino MG, Magni E, Massacesi C, et al: First clinical experience of orally active epidermal growth factor receptor inhibitor combined with simplified FOLFOX6 as first-line treatment for metastatic colorectal cancer. Cancer 110: 752-758, 2007. 
20. Velcheti V, Morgensztern D and Govindan R: Management of patients with advanced non-small cell lung cancer: role of gefitinib. Biologics 4: 83-90, 2010.

21. Rho JK, Choi YJ, Jeon BS, et al: Combined treatment with silibinin and epidermal growth factor receptor tyrosine kinase inhibitors overcomes drug resistance caused by T790M mutation. Mol Cancer Ther 9: 3233-3243, 2010.

22. Janjigian YY, Azzoli CG, Krug LM, et al: Phase I/II trial of cetuximab and erlotinib in patients with lung adenocarcinoma and acquired resistance to erlotinib. Clin Cancer Res (In press).

23. Giustina A, Mazziotti G and Canalis E: Growth hormone, insulin-like growth factors, and the skeleton. Endocr Rev 29: 535-559, 2008

24. Burrow S, Andrulis IL, Pollak M and Bell RS: Expression of insulin-like growth factor receptor, IGF-1, and IGF-2 in primary and metastatic osteosarcoma. J Surg Oncol 69: 21-27, 1998.

25. Scotlandi K, Benini S, Sarti M, et al: Insulin-like growth factor I receptor-mediated circuit in Ewing's sarcoma/peripheral neuroectodermal tumor: a possible therapeutic target. Cancer Res 56: 4570-4574, 1996.

26. Dong J, Demarest SJ, Sereno A, et al: Combination of two insulin-like growth factor-I receptor inhibitory antibodies targeting distinct epitopes leads to an enhanced antitumor response. Mol Cancer Ther 9: 2593-2604, 2010.
27. Yakar S, Courtland HW and Clemmons D: IGF-1 and bone: new discoveries from mouse models. J Bone Miner Res 25 2267-2276, 2010

28. Wang Y, Hailey J, Williams $\mathrm{D}$, et al: Inhibition of insulin-like growth factor-I receptor (IGF-IR) signaling and tumor cell growth by a fully human neutralizing anti-IGF-IR antibody. Mol Cancer Ther 4: 1214-1221, 2005.

29. Ciampolillo A, De Tullio C and Giorgino F: The IGF-I/IGF-I receptor pathway: implications in the pathophysiology of thyroid Cancer. Curr Med Chem 12: 2881-2891, 2005.

30. Grimberg A: Mechanisms by which IGF-I may promote cancer. Cancer Biol Ther 2: 630-635, 2003.

31. Shih YW, Chien ST, Chen PS, Lee JH, Wu SH and Yin LT: Alpha-mangostin suppresses phorbol 12-myristate 13-acetateinduced MMP-2/MMP-9 expressions via alphavbeta3 integrin/ FAK/ERK and NF-kappaB signaling pathway in human lung adenocarcinoma A549 cells. Cell Biochem Biophys 58: 31-44, 2010.

32. Zaoui P, Cantin JF, Alimardani-Bessette M, et al: Role of metalloproteases and inhibitors in the occurrence and progression of diabetic renal lesions. Diabetes Metab 26 (Suppl. 4): 25-29, 2000 . 